\title{
Persistent Atrazine Toxicity in Mohave Desert
} Shrub Communities

\author{
RICHARD HUNTER, A. WALLACE AND E. M. ROMNEY
}

Highlight: Atrazine (11.2kg/ha active ingredient) was applied in 1967 and 1968 to three areas of the northern Mohave Desert to destroy perennial shrub cover. Of the 23 perennial species 12 were completely eliminated. Two species, Mohave Yucca (Yucca schidigera) and big galleta (Hilaria rigida) showed no effects. Plants of creosote bush (Larrea tridentata), Nevada Ephedra (Ephedra nevadensis), and range ratany (Krameria parvifolia) were severely damaged but many survived through crown sprouting. Scattered plants of the invading species shadscale (Atriplex confertifolia), desertalyssum (Lepidium fremontii), and Russian thistle (Salsola paulsenii) became established by 1975 on the fertile mounds under killed shrubs. Glasshouse tests of seedling survival on soils sampled eight years after treatment showed 65 to 95\% mortality, as compared to 3 to $8 \%$ mortality on control soils.

For several decades, activities which damage the perennial vegetation and soil surface of the Mohave Desert have been rapidly expanding. Prominent examples are road construction, power line installation, weapons testing and use of off-road vehicles. In addition the vegetation is subject to grazing pressure from burros, rabbits, and smaller rodents, and a varied insect population.

The vegetative cover plays an important role in dust suppression, penetration of rainfall, and reduction of runoff, and also supports a complex and unique ecosystem. Knowledge of how this vegetation recovers from damage is important for determination of suitable land use practices.

This study was part of efforts to determine the long-term response of Mohave Desert shrub communities to denudation (Wallace and Romney 1974, 1976). The study area is dominated by long-lived shrubs covering $10-30 \%$ of

\footnotetext{
Authors are post-doctoral plant physiologist, professor of plant physiology, and research soil scientist, Laboratory of Nuclear Medicine and Radiation Biology, University of California, Los Angeles, Dr. Hunter's address is P.O. Box 495, Mercury, Nevada 89023.

Research was conducted under contract EY-76-C-03-0012 between the University of California and the U.S. Energy Research and Development Administration. Field Support was provided by the Civil Effects Test Organization, USERDA.

Manuscript received August 11, 1977.
}

the surface. Mounds of fertile soil have formed under the shrubs. These "fertile islands" (Muller and Muller 1956) support the growth of winter annuals and are a favored microhabitat for establishment of shrub seedlings. It was postulated that denudation without disturbing these "fertile islands" would accelerate the revegetation process. Atrazine trials were initiated to investigate its ability to remove desert vegetation without disturbing these important soil surface properties.

\section{Methods}

Atrazine $(11.2 \mathrm{~kg} / \mathrm{ha}$ active ingredient: $10 \%$ granules) was broadcast in February 1967 onto a single $930-\mathrm{m}^{2}$ plot spanning a wash. From 1968 to 1973 the drainage channel of this wash received tertiary stage water from sewage oxidation ponds from the town of Mercury, Nevada (Wallace and Romney 1972, pp. 315324). In May 1968 two nearby circular plots $\left(730 \mathrm{~m}^{2}\right.$ each) were treated similarly with atrazine. Adjacent plots received unrelated treatments.

One of the two circular plots was sprinkler-irrigated during the summers of $1968(23 \mathrm{~cm}), 1969(20 \mathrm{~cm})$, and $1970(34 \mathrm{~cm})$, as was a control plot. All plots received natural precipitation, which by calendar year from 1968-1975 was $11,20,8,8,17,18,11$, and $8 \mathrm{~cm}$.

The wash plots were abandoned, but all live perennial plants on the circular plots were censused in 1968; i.e. each plant's position, species, height, and two perpendicular widths were recorded.

Toxicity symptoms on Russian thistle (Salsola paulsenii) plants on the treated plots were noted in 1975, and the unexpected persistence led to an evaluation of the herbicide effects. The two circular plots were recensused in August 1975. Positions, species and sizes of all live and dead shrubs and Russian thistle plants were recorded. Dead winter annuals were counted, harvested, and weighed from fifteen $0.1-\mathrm{m}^{2}$ areas on fertile islands of these plots and two control plots. Perennials on the control plots had been censused in November 1974 for an unrelated experiment. A search of the control plots for Russian thistle in September 1975 was unsuccessful.

The wash plot was censused in December 1975 through the use of four $100-\mathrm{m}^{2}$ belt transects, with species and sizes of all live and dead plants recorded. The topographies, plant communities and treatments of adjacent plots along the wash differed from those of 
the atrazine plot, hence no suitable control area was found to census.

Shrub biomass (B, kg) was determined from the size measurements. A volume $\left(\mathrm{V}, \mathrm{m}^{3}\right)$ was calculated using an assumed cylindric shape, and this was fitted to a regression line of $B \mathrm{vs} V$, previously determined by harvest of plants outside the plot areas. Previously published regression lines (Romney et al. 1973) were modified for creosotebush (Larrea tridentata) to $\mathrm{B}=1.17 \mathrm{~V}^{1 / 2}$ and for Indian ricegrass (Oryzopsis hymenoides) to $\mathrm{B}=0.248 \mathrm{~V}$. New lines were generated for Mohave Yucca (Yucca schidigera, B = $\left.7.83 \mathrm{~V}^{1.25}, \mathrm{r}=.99\right)$ and Russian thistle $(\mathrm{B}=2.61 \mathrm{~V}+.02, \mathrm{r}=.98)$. Biomass for species without lines was calculated using lines for species of similar growth habit.

Importance values (I) were calculated for each species as the sums of relative frequency, percent of total biomass, and percent of total cover. A species occurring as a monoculture would have an I of 300 , which would decrease with increasing species diversity. Relative frequency was determined on the circular plots by dividing the position data into $10^{\circ}$ wedges and calculating the percent of wedges occupied.

In December 1975, soil samples were taken from three sites beneath shrubs ("fertile islands") and from three adjacent bare areas on each of the three atrazine treated plots and two nonirrigated control plots. Each sample was divided into surface soil $(0-7.5 \mathrm{~cm})$ and subsoil $(7.5-15 \mathrm{~cm})$ and placed in a 6 -inch pot in an unheated glasshouse. Each pot contained $3-4 \mathrm{~kg}$ soil. Initial seeding with red brome (Bromus rubens) in December 1975 produced too few seedlings for valid comparison of atrazine activities. In December 1976 the pots were resown with 10 seeds each of hairy-leaved caulanthus (Caulanthus lasiophyllus var. utahensis) and fertilized with $75 \mathrm{ml}$ of $0.05 \mathrm{M} \mathrm{KNO}_{3}$. Survival of these plants and volunteers (primarily red brome) was followed for 75 days. Trays were placed under all pots to prevent drainage loss, and all water added was distilled.

Species nomenclature is from Beatley (1976). Common names are from a variety of sources.

\section{Results}

Toxicity symptoms and defoliation began soon after application of atrazine. Only Mohave yucca and big galleta (Hilaria rigida) showed no effects. By February 1969 all winterfat (Ceratoides lanata) and desert globemallow (Sphaeralcea ambigua) were dead.

Irrigation accelerated the atrazine effects on several species. All but two of 136 Shockley's goldenhead (Acamptopappus shockleyi) were dead on the irrigated plot by February 1969, but on the dry plot most leafed out that spring, then died before flowering. Bur-sage (Ambrosia dumosa) produced leaves and a few flower buds in the spring of 1969 on the dry plot, but just a few leaves on the irrigated plot. All 34 shadscale plants (Atriplex confertifolia) on the irrigated plot appeared dead by October 1969 , but only 1 of 14 on the dry plot did.

In October 1969 most creosotebush were alive but without green leaves; all but one desert thorn (Lycium andersonii) and all but two Nevada Ephedra (Ephedra nevadensis) were dead; and most range ratany (Krameria parvifolia) were alive but dormant. Indian ricegrass (Oryzopsis hymenoides) survived, and big galleta grew well, flowering both spring and fall of 1969 .

Sporadic visits to the plots from 1970-1975 were made, observers noting a near total absence of recovery.

The population characteristics of the circular atrazine and control plots, as measured in 1968 and 1975, are summarized in Tables 1 and 2. Several common Mohave Desert species were completely eliminated, including Shockley's goldenhead, bursage, Death Valley Ephedra (Ephedra funerea), desert thorn, Mohave aster (Machaeranthera tortifolia), and desert globemallow. These include

Table 1. Shrub population characteristics of atrazine plots in 1968 and 1975 . Values are averages for one irrigated and one dry plot, covering $1,459 \mathrm{~m}^{2}$.

\begin{tabular}{|c|c|c|c|c|c|c|c|c|c|}
\hline \multirow{3}{*}{ Species } & \multicolumn{4}{|c|}{1968} & \multicolumn{5}{|c|}{1975} \\
\hline & \multirow{2}{*}{$\begin{array}{c}\text { Live } \\
\text { density } \\
\# / 100 \mathrm{~m}^{2}\end{array}$} & \multirow{2}{*}{$\begin{array}{c}\text { Biomass } \\
\mathrm{kg} / \mathrm{ha}\end{array}$} & \multirow{2}{*}{$\begin{array}{c}\text { Cover } \\
\%\end{array}$} & \multirow[t]{2}{*}{$I^{d}$} & \multicolumn{2}{|c|}{$\# / 100 \mathrm{~m}^{2}$} & \multirow{2}{*}{$\begin{array}{c}\text { Biomass } \\
\mathrm{kg} / \mathrm{ha}\end{array}$} & \multirow{2}{*}{$\begin{array}{c}\text { Cover } \\
\%\end{array}$} & \multirow[t]{2}{*}{ I } \\
\hline & & & & & Live & Dead $^{a}$ & & & \\
\hline Acamptopappus shockleyi (Shockley's Goldenhead) & 18.2 & 12 & 1.0 & 89 & 0 & 14.5 & 0 & 0 & 0 \\
\hline Ambrosia dumosa (bur-sage) & 27.7 & 460 & 5.6 & 133 & 0 & 25.3 & 0 & 0 & 0 \\
\hline Atriplex confertifolia (shadscale) & 3.3 & 69 & 0.4 & 47 & 1.4 & 2.5 & 82 & 0.3 & 30 \\
\hline Cacti & 0.8 & 66 & 0.1 & 12 & 0.8 & 0.1 & 51 & 0.2 & 16 \\
\hline Ceratoides lanata (winterfat) & 1.8 & 11 & 0.1 & 30 & 0.1 & 1.2 & 0 & 0.0 & 1 \\
\hline Ephedra funerea (Death Valley ephedra) & 3.6 & 165 & 1.5 & 39 & 0 & 1.7 & 0 & 0 & 0 \\
\hline Ephedra nevadensis (Nevada ephedra) & 3.2 & 93 & 1.4 & 44 & 0.3 & 2.6 & 9 & 0.5 & 14 \\
\hline Hilaria rigida (big galleta) ${ }^{b}$ & 3.2 & 0 & 0.0 & 6 & 3.1 & 0.1 & 0 & 0.1 & 7 \\
\hline Krameria parvifolia (range ratany) & 8.4 & 78 & 2.2 & 80 & 1.0 & 6.6 & 6 & 0.2 & 19 \\
\hline Larrea tridentata (creosotebush) & 4.4 & 135 & 1.3 & 58 & 0.6 & 3.7 & 8 & 0 & 7 \\
\hline Lepidium fremontii (desert-alyssum) & 0.7 & 1 & 0.0 & 14 & 1.9 & 0.1 & 48 & 0.3 & 29 \\
\hline Lycium andersonii (desert-thorn) & 5.3 & 239 & 2.4 & 78 & 0 & 5.3 & 0 & 0 & 0 \\
\hline Machaeranthera tortifolia (Mohave aster) & 4.5 & 5 & 0.2 & 44 & 0 & 2.7 & 0 & 0 & 0 \\
\hline Oryzopsis hymenoides (Indian ricegrass) & 3.2 & 7 & 0.0 & 34 & 0.7 & 2.4 & 2 & 0.0 & 13 \\
\hline Sphaeralcea ambigua (desert globemallow) & 5.8 & 3 & 0.2 & 55 & 0 & 2.9 & 0 & 0 & 0 \\
\hline Stephanomeria pauciflora (desert wirelettuce) ${ }^{\mathbf{b}}$ & 0 & 0 & 0 & 0 & 0.1 & 0 & 4 & 0.0 & 2 \\
\hline Yucca schidigera (Mohave yucca) & 1.9 & 3977 & 2.6 & 118 & 1.9 & 0.1 & 9381 & 4.4 & 197 \\
\hline Other species ${ }^{c}$ & 0.7 & 15 & 0.3 & 13 & 0 & 0.6 & 0 & 0 & 0 \\
\hline Salsola paulsenii (Russian thistle) & - & - & - & - & 13.8 & - & 168 & 0.4 & 74 \\
\hline $\begin{array}{l}\text { Totals } \\
\text { Simpson Index }\end{array}$ & 96.6 & $\begin{array}{r}5335 \\
\quad .08\end{array}$ & $6^{19.3}$ & 894 & 25.7 & 72.3 & $\begin{array}{c}9818 \\
.286\end{array}$ & 6.4 & 405 \\
\hline
\end{tabular}

a Missing plants (present in 1968 but not in 1975) totaled $14.7 / 100 \mathrm{~m}^{2}$, and may be added to the dead plant values.

${ }^{\mathrm{b}} \mathrm{A}$ species for which a biomass regression line was not available; lines for species of similar habit were used.

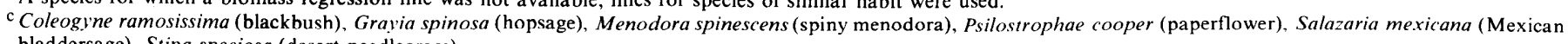
bladdersage), Stipa speciosa (desert needlegrass).

dImportance values 
Table 2. Population characteristics of control plots $\left(1,459 \mathrm{~m}^{2}\right)$ in 1968 and 1974 . Values are averages for one irrigated and one dry plot.

\begin{tabular}{|c|c|c|c|c|c|c|c|c|c|}
\hline \multirow{3}{*}{ Species } & \multicolumn{4}{|c|}{1968} & \multicolumn{5}{|c|}{1974} \\
\hline & \multirow{2}{*}{$\begin{array}{c}\text { Live } \\
\text { density } \\
\# / 100 \mathrm{~m}^{2}\end{array}$} & \multirow{2}{*}{$\begin{array}{c}\text { Biomass } \\
\mathrm{kg} / \mathrm{ha}\end{array}$} & \multirow{2}{*}{$\begin{array}{c}\text { Cover } \\
\%\end{array}$} & \multirow{2}{*}{$I^{d}$} & \multicolumn{2}{|c|}{$\# / 100 \mathrm{~m}^{2}$} & \multirow{2}{*}{$\begin{array}{c}\text { Biomass } \\
\mathrm{kg} / \mathrm{ha}\end{array}$} & \multirow{2}{*}{$\begin{array}{c}\text { Cover } \\
\%\end{array}$} & \multirow{2}{*}{ I } \\
\hline & & & & & Live & Dead $^{a}$ & & & \\
\hline Acamptopappus shockleyi (Shockley's goldenhead) & 23.4 & 19 & 2.2 & 90 & 28.2 & 3.6 & 142 & 2.2 & 95 \\
\hline Ambrosia dumosa (bur-sage) & 17.2 & 254 & 3.0 & 104 & 18.4 & 0.4 & 401 & 4.4 & 106 \\
\hline Atriplex confertifolia (shadscale) & 1.4 & 8 & 0.0 & 21 & 0.2 & 1.2 & 7 & 0.0 & 4 \\
\hline Cactib & 0.4 & 3 & 0.2 & 10 & 0.6 & 0.1 & 38 & 0.1 & 14 \\
\hline Ceratoides lanata (winterfat) & 63.8 & 270 & 2.9 & 115 & 31.5 & 6.9 & 474 & 3.2 & 114 \\
\hline Ephedra funerea (Death Valley ephedra) & 2.0 & 216 & 1.5 & 47 & 2.1 & 0 & 385 & 1.9 & 51 \\
\hline Ephedra nevadensis (Nevada ephedra) & 2.1 & 61 & 0.8 & 28 & 2.4 & 0 & 112 & 1.2 & 32 \\
\hline Hilaria rigida (big galleta) ${ }^{\mathrm{b}}$ & 0 & 0 & 0 & 0 & 0 & 0 & 0 & 0 & 0 \\
\hline Krameria parvifolia (range ratany) & 7.3 & 83 & 2.0 & 75 & 7.3 & 0.2 & 92 & 2.3 & 69 \\
\hline Larrea tridentata (creosotebush) & 5.6 & 363 & 3.5 & 88 & 5.8 & 0.1 & 392 & 3.9 & 82 \\
\hline Lepidium fremontii (desert-alyssum) & 0.1 & 0 & 0 & 3 & 2.0 & 0.1 & 9 & 0.0 & 25 \\
\hline Lycium andersonii (desert-thorn) & 6.7 & 319 & 3.0 & 89 & 6.7 & 0.1 & 400 & 3.8 & 87 \\
\hline Machaeranthera tortifolia (Mohave aster) & 0 & 0 & 0 & 0 & 0.3 & 0.2 & 1 & 0.0 & 3 \\
\hline Oryzopsis hymenoides (Indian ricegrass) & 1.6 & 4 & 0.0 & 22 & 0.7 & 0.8 & 0 & 0.0 & 11 \\
\hline Sphaeralcea ambigua (desert globemallow) & 9.5 & 3 & 0.2 & 57 & 2.6 & 9.7 & 3 & 0.2 & 38 \\
\hline Stephanomeria pauciflora (desert wirelettuce) ${ }^{b}$ & 0.1 & 13 & 0.0 & 2 & 0.1 & 0 & 4 & 0.0 & $\begin{array}{r}30 \\
2\end{array}$ \\
\hline Yucca schidigera (Mohave yucca) & 0.6 & 1534 & 0.8 & 50 & 0.6 & 0.1 & 2842 & 1.2 & 68 \\
\hline Other species ${ }^{c}$ & 6.8 & 122 & 1.1 & 71 & 8.7 & 0.3 & 228 & 2.1 & 82 \\
\hline Salsola paulsenii (Russian thistle) & - & - & - & - & 0 & 0 & 0 & 0 & 0 \\
\hline Totals & 145.6 & 3299 & 21.2 & 872 & 114.6 & 23.6 & 5528 & 26.1 & 883 \\
\hline Simpson Index & \multicolumn{4}{|c|}{.086} & & & .084 & & \\
\hline
\end{tabular}

${ }^{a}$ Missing plants totaled $7.3 / 100 \mathrm{~m}^{2}$

${ }^{\mathrm{b}} \mathrm{A}$ species for which a biomass regression line was not available; lines for species of similar habit were used.

c Coleogyne ramosissima (blackbush), Grayia spinosa (hopsage), Menodora spinescens (spiny menodora).Psilostrophae cooper (papcrflower). Salazaria mexicana (Mcxican bladdersage), Stipa speciosa (desert needlegrass).

dimportance values

three of the five species with importance values above 80 . Most shrub species declined in importance, the species with increased importance value being beaver-tail cactus (Opuntia basilaris), pencil cactus (Opuntia ramosissima), big galleta, the wash and roadside shrub desert wirelettuce (Stephanomeria pauciflora), desert alyssum (Lepidium fremontii), and Mohave yucca. Russian thistle, a summer annual, is included in Tables 1 and 2 because its growth habits resemble those of perennial shrubs rather than other annuals, which are primarily ephemerals restricted to winter and spring growth (Beatley 1965).

Diversity, as measured by the Simpson index, decreased markedly on the atrazine plots (Tables 1 and 2). Cover and frequency of plants were strongly decreased, but lost biomass was more than compensated for by increased growth of Mohave Yucca. The few new plants all appeared on fertile islands, which remained intact in 1975 in spite of the loss of cover and sources of litter.

As atrazine is a photosynthetic inhibitor (Shimabukuro et al. 1976), one might expect larger plants, with greater carbohydrate reserves, to survive longer. This did not appear to be the case (Table 3), as surviving plants ranged considerably in size and their average size did not differ significantly $(p=.05)$ from those killed. Survival depended strongly on species, and was decreased by irrigation. Average size of plants present at both censuses increased in

Table 3. Characteristics of shrubs surviving atrazine treatment. Atrazine $(11.2 \mathrm{~kg} / \mathrm{ha})$ was applied February 1968 , irrigation was on one plot during summers of 1968, 1969 and 1970, and final size was measured in August 1975.

\begin{tabular}{|c|c|c|c|c|c|c|c|c|}
\hline Species & $\begin{array}{l}\text { Irrigation } \\
\text { treatment }\end{array}$ & $\begin{array}{l}\text { Number } \\
\text { treated }\end{array}$ & $\begin{array}{c}\% \\
\text { Surviving }\end{array}$ & $\begin{array}{c}\text { Average } \\
\text { initial size } \\
\text { of survivors } \\
\mathrm{g} \\
\end{array}$ & $\begin{array}{c}\% \text { change in } \\
\text { average size } \\
1968-1975\end{array}$ & $\begin{array}{c}\text { Average } \\
\text { initial size } \\
\text { non-survivors } \\
\mathrm{g} \\
\end{array}$ & $\begin{array}{l}\text { New } \\
\text { plants }\end{array}$ & $\begin{array}{c}\text { Final size } \\
\text { of new } \\
\text { plants } \\
\mathrm{g}\end{array}$ \\
\hline $\begin{array}{l}\text { Atriplex } \\
\quad \text { confertifolia }\end{array}$ & $\begin{array}{l}\text { Irrigated } \\
\text { Dry }\end{array}$ & $\begin{array}{l}34 \\
14\end{array}$ & $\begin{array}{l}12 \\
21\end{array}$ & $\begin{array}{r}755 \\
88\end{array}$ & $\begin{array}{r}207 \\
1471\end{array}$ & $\begin{array}{l}195 \\
154\end{array}$ & $\begin{array}{l}9 \\
4\end{array}$ & $\begin{array}{l}68 \\
70\end{array}$ \\
\hline Cactus spp. & $\begin{array}{l}\text { Irrigated } \\
\text { Dry }\end{array}$ & $\begin{array}{l}3 \\
8\end{array}$ & $\begin{array}{r}67 \\
100\end{array}$ & $\begin{array}{l}263 \\
294\end{array}$ & $\begin{array}{l}47 \\
59\end{array}$ & $\begin{array}{c}287 \\
-\end{array}$ & $\begin{array}{l}1 \\
1\end{array}$ & $\begin{array}{r}26 \\
210\end{array}$ \\
\hline $\begin{array}{l}\text { Ephedra } \\
\quad \text { nevadensis }\end{array}$ & $\begin{array}{l}\text { Irrigated } \\
\text { Dry }\end{array}$ & $\begin{array}{l}18 \\
27\end{array}$ & $\begin{array}{r}6 \\
15\end{array}$ & $\begin{array}{l}221 \\
132\end{array}$ & $\begin{array}{l}-27 \\
-2\end{array}$ & $\begin{array}{l}137 \\
218\end{array}$ & $\begin{array}{l}0 \\
0\end{array}$ & - \\
\hline $\begin{array}{l}\text { Hilaria } \\
\quad \text { rigida }\end{array}$ & $\begin{array}{l}\text { Irrigated } \\
\text { Dry }\end{array}$ & $\begin{array}{r}7 \\
40\end{array}$ & $\begin{array}{r}100 \\
92\end{array}$ & $\begin{array}{r}35 \\
6\end{array}$ & $\begin{array}{r}9 \\
106\end{array}$ & - & $\begin{array}{l}0 \\
1\end{array}$ & $\overline{2}$ \\
\hline $\begin{array}{l}\text { Krameria } \\
\quad \text { parvifolia }\end{array}$ & $\begin{array}{l}\text { Irrigated } \\
\text { Dry }\end{array}$ & $\begin{array}{l}88 \\
32\end{array}$ & $\begin{array}{r}3 \\
34\end{array}$ & $\begin{array}{l}118 \\
143\end{array}$ & $\begin{array}{r}5 \\
-53\end{array}$ & $\begin{array}{l}102 \\
132\end{array}$ & $\begin{array}{l}0 \\
0\end{array}$ & - \\
\hline $\begin{array}{l}\text { Larrea } \\
\quad \text { tridentata }\end{array}$ & $\begin{array}{l}\text { Irrigated } \\
\text { Dry }\end{array}$ & $\begin{array}{l}22 \\
42\end{array}$ & $\begin{array}{r}0 \\
21\end{array}$ & $-\overline{435}$ & $\overline{-47}$ & $\begin{array}{l}194 \\
454\end{array}$ & $\begin{array}{l}0 \\
0\end{array}$ & - \\
\hline $\begin{array}{l}\text { Yucca } \\
\quad \text { schidigera }\end{array}$ & $\begin{array}{l}\text { Irrigated } \\
\text { Dry }\end{array}$ & $\begin{array}{l}10 \\
18\end{array}$ & $\begin{array}{r}80 \\
100 \\
\end{array}$ & $\begin{array}{l}31,303 \\
18,147 \\
\end{array}$ & $\begin{array}{l}90 \\
87\end{array}$ & 180 & $\begin{array}{l}1 \\
1\end{array}$ & $\begin{array}{r}1 \\
107\end{array}$ \\
\hline
\end{tabular}


Table 4. Dead winter annuals in shrub clumps of circular plots August 1975ª.

\begin{tabular}{|c|c|c|c|c|c|c|}
\hline \multirow[b]{2}{*}{ Treatment } & \multicolumn{2}{|c|}{ Bromus rubens } & \multicolumn{2}{|c|}{ Festuca octoflora } & \multicolumn{2}{|c|}{ Other species $b$} \\
\hline & No. $/ m^{2} \times \mathrm{se}$ & $\mathrm{g} / \mathrm{m}^{2} \pm \mathrm{se}$ & No. $/ \mathrm{m}^{2} \pm \mathrm{se}$ & $\mathrm{g} / \mathrm{m}^{2} \pm \mathrm{se}$ & No. $/ \mathrm{m}^{2} \pm \mathrm{se}$ & $\mathrm{g} / \mathrm{m}^{2} \pm \mathrm{se}$ \\
\hline Control & $93 \pm 58$ & $15 \pm 9$ & $107 \pm 57$ & $2 \pm 1$ & $89 \pm 26$ & $8 \pm 2$ \\
\hline $\begin{array}{c}\text { Control/Irrigated } \\
1968-1970\end{array}$ & $1045 \pm 335$ & $44 \pm 12$ & $141 \pm 98$ & $2 \pm 1$ & $20 \pm 6$ & $3 \pm 1$ \\
\hline Atrazine $11.2 \mathrm{~kg} / \mathrm{ha}$ & $9 \pm 3$ & $1.2 \pm 0.6$ & $5 \pm 2$ & $0.4 \pm 0.2$ & $1 \pm 1$ & $0.0 \pm 0.0$ \\
\hline $\begin{array}{c}\text { Atrazine/Irrigated } \\
1968-1970\end{array}$ & $10 \pm 4$ & $6 \pm 2$ & $149 \pm 60$ & $14 \pm 5$ & $3 \pm 2$ & $0.1 \pm 0.1$ \\
\hline
\end{tabular}

a Shrubs cover 15-30\% of the soil surface (Wallace and Romney 1972, p. 230). Winter annuals in bare areas are rare, and were not censused.

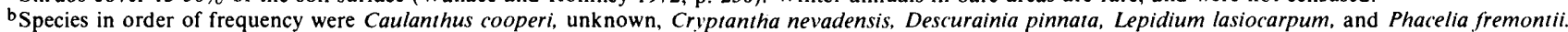

four of the seven species with survivors, indicative of severe and continuing damage to individuals of the other three species.

Dominant winter annuals are red brome and six-weeks fescue (Festuca octoflora), which are both killed by atrazine. Table 4 shows the expected marked decrease in winter annual production on the atrazine plots. Six-weeks fescue on the irrigated plot occurred in numbers similar to those on control plots, but its biomass was greater.

On the wash plot several damaged species fared better than on the circular plots, showing regrowth from stems rather than crowns. Big galleta invaded the wash channel during the years when it received extra water, but most was dead in 1975, 2 years after the water was diverted. Shrub mortality was lower on slopes within a few meters of the wash channel (Table 5).

Table 5. Average plant cover, weight, and live and dead plants in $100 \mathrm{~m}^{2}$ transects on the atrazine-treated wash plot, August 1975.

\begin{tabular}{lcccccc}
\hline & $\begin{array}{c}\text { Distance from } \\
\text { wash center }\end{array}$ & $\begin{array}{c}\text { Cover } \\
\text { m }\end{array}$ & $\%$ & $\begin{array}{c}\text { Average plant } \\
\text { weight }\end{array}$ & \multicolumn{2}{c}{ Plants/100m 2} \\
\cline { 5 - 7 } & $0-3$ & 27 & 1414 & $41^{\mathrm{a}}$ & $86^{\mathrm{a}}$ \\
\hline In main wash & $0-3$ & & Live & Dead \\
South side of wash & $3-8$ & 16 & 556 & 30 & 58 \\
Bajada & $9-13$ & 11 & 289 & 39 & 34 \\
Bajada & $13-17$ & 5 & 126 & 26 & 68 \\
\hline
\end{tabular}

${ }^{\text {a }}$ This transect includes 21 live and 54 dead big galleta (Hilaria rigida), a species which was unaffected by atrazine

The seedling survival test, to detect residual atrazine toxicity, showed there was sufficient activity left in 1975 to kill nontolerant seedlings. Figure 1 shows seedling mortality began 2 weeks after germination, after exhaustion of food reserves in the seed. Death followed severe tip burn. Survival in control soils was 92 to $97 \%$ after 75 days, but averaged only $5-35 \%$ on treated soils (Table 6). Survival was

Table 6. Seedling survival to day 75 in potted soils from atrazine plots.

\begin{tabular}{lccc}
\hline & $\begin{array}{c}\text { Number } \\
\text { of pots }\end{array}$ & $\begin{array}{c}\text { Average } \\
\text { \% survival } \\
\pm \text { sem }\end{array}$ & $\begin{array}{c}\text { Probability } \\
\text { survival=control }\end{array}$ \\
\hline Control Plot 8 & 12 & $91 \pm 3$ & - \\
Control Rock Valley & 12 & $95 \pm 2$ & - \\
Atrazine Plot 21 & 12 & $34 \pm 13$ & $<.001$ \\
$\quad 12$ & $19 \pm 9$ & $<.001$ \\
$\quad$ Plot 22 & 12 & $4 \pm 2$ & $<.001$ \\
Wash & 12 & $90 \pm 3 \mathrm{a}$ & - \\
Barub Control & 12 & $96 \pm 2$ & - \\
Shrub Atrazine & 18 & $30 \pm 9 \mathrm{a}$ & $<.001$ \\
Bare Atrazine & 18 & $7 \pm 6$ & $<.001$ \\
\hline
\end{tabular}

${ }^{a}$ On control soils $p<.10$ that $\%$ survival on shrub and bare are equal, while on atrazine treated soils $\mathrm{p}<.025$. better in soil from beneath shrubs than in soil from bare areas. There was no significant difference $(p=.05)$ between survival rates on surface and subsurface soils.

\section{Discussion}

Residual atrazine toxicity was apparent more than 8 years after application to Mohave Desert soils. In comparison, atrazine applied to agricultural soils has rarely been reported to persist at phytotoxic levels longer than 2 years (Birk and Roadhouse 1964; Fink and Fletchall 1969; Buchanan and Hiltbold 1973; Ritter et al. 1974; Best et al. 1975). Treatment of natural vegetation in less arid regions has been followed by invasion of susceptible species within 1 year (Dowler et al. 1968; Eckert 1974), or has required repeat applications for continued effectiveness (Houston 1977).

Persistence of atrazine may be only partially explained by the high rate applied and granular formulation. Recent work (Kaufman and Kearney 1970; Skipper and Volk 1972; Best 1974) suggests that hydrolysis to hydroxyatrazine should be the primary mode of detoxication at the $\mathrm{pH}$ of 8 to 9 prevailing in these soils (Romney et al. 1973). In contrast, the greater residual toxicity in bare area soils suggests the low organic matter content, averaging $0.4-0.6 \%$ organic $C$, might reduce microbial detoxication (Harris et al. 1969; Roeth et al. 1969; Hance 1973, 1974; Nikolova 1973) in comparison to shrub soils, whose surface layers contain 1 to $3 \%$ organic $\mathrm{C}$.

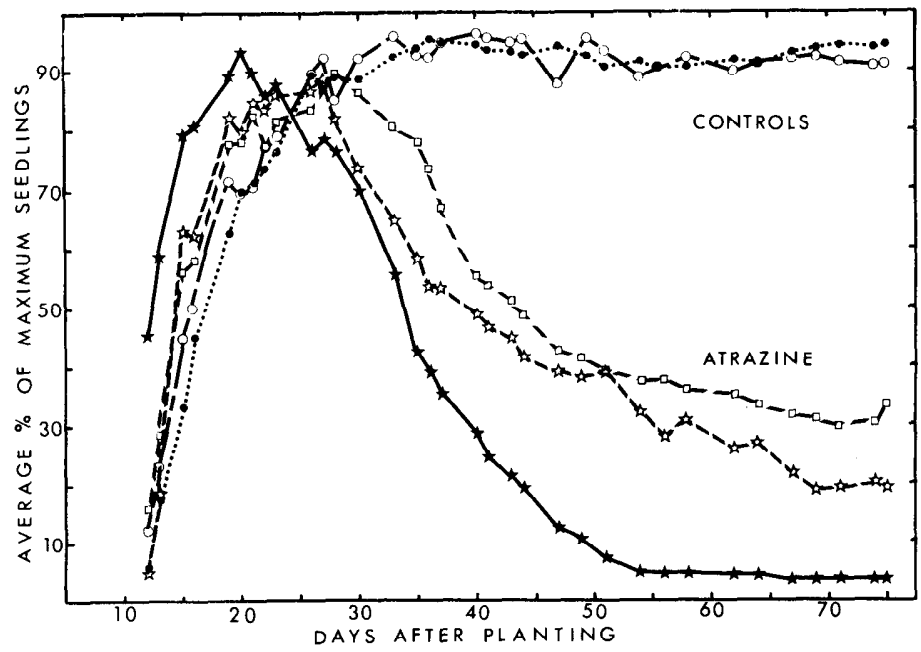

Fig. 1. Survival of seedlings germinating in soils taken from atrazinetreated and control plots. For error estimates on day 75 see Table 6. Symbols for individual plots are: $\bullet$ Mercury dry control plot; 0 Rock Valley soil; $\nmid$ Mercury wash atrazine plot; $\square$ Mercury irrigated atrazine plot; \&Mercury dry atrazine plot. 
Though desert soils are more frequently "dry" than those of more mesic areas, they are not air dry. In Rock Valley, 24 $\mathrm{km}$ from these plots, soil water potentials at $15 \mathrm{~cm}$ were greater than -30 bars for 31 of 48 reporting periods in 1973, 26 of 41 in 1974, and 16 of 38 in 1975; soil temperatures from January through April, the months soil is most often wet, ranged from 4 to $25^{\circ} \mathrm{C}$ (Bamberg et al. 1974; Turner et al. 1975; Maza and Turner 1976). Desert plants have a net carbon uptake at potentials above approximately -50 bars (Bamberg et al. 1975) and presumably chemical hydrolysis would not be inhibited at that potential, but atrazine decomposing microbes might be.

Birk and Roadhouse (1964) found that atrazine stored in air dry soil lost $60 \%$ of extractable activity in 2 years. Given a similar rate of loss $1.3 \mathrm{~kg} /$ ha would remain on our plots after 8 years. However, given the considerably wetter Mohave soils, we feel lack of moisture is not primarily responsible for the persistence.

It therefore appears more likely that the rate-limiting step for atrazine detoxication under Mohave conditions is microbial degradation. Microbial decomposition may be inhibited by the combination of low organic $\mathrm{C}$, high $\mathrm{pH}$, low moisture content, and frequently extreme surface temperatures.

Shrub survival and invasion of treated areas appears to be an interaction of several factors. Invading species appear to have found microsites of reduced toxicity. Some shrub species had a small percentage of survivors due primarily to crown sprouting.

Size did not appear related to survival, but location near a wash did. The latter observation may be due to greater wetting and rooting depths near the wash, and perhaps atrazine loss with runoff from the wash slopes. Soil wetting depths rarely exceed $60 \mathrm{~cm}$ on bajadas, but would have been much deeper in the wash, with correspondingly deeper rooting. Atrazine may not have penetrated to the majority of the roots in the wash, or may have been diluted by the larger soil volume available.

Survival may also be related to growth habit. The most severely affected species are either drought deciduous or suffrutescent, while most survivors maintain leaves all summer (creosotebush, shadscale, and Mohave Yucca) or have greatly reduced leaves (cacti, ephedra). Range ratany loses leaves in winter, but is not drought deciduous. The drought deciduous shrubs have high photosynthetic rates, but only for a short time each year (Bamberg et al. 1975), and may be more severely damaged by photsynthetic inhibitors.

The physiology of photosynthesis may be important. Wallace et al. (1971) report ratios of RUDP carboxylase to PEP carboxylase activity of several Mohave Desert species. Of the seven species found on these plots, four species with ratios less than two (indicating $\mathrm{C}_{3}$ or CAM photosynthesis) had some survivors, while three with no survivors had ratios greater than three (indicating $\mathrm{C}_{4}$ photosynthesis).

\section{Literature Cited}

Bamberg, S. A., H. Kaaz, B. Maza, and F. B. Turner. 1974. Abiotic. p. 11-24. In: F. B. Turner (ed.), Rock Valley validation site report. US/IBP Desert Biome Res. Memo. 74-2.
Bamberg, S. A., G. E. Kleinkopf, A. Wallace, and A. Vollmer. 1975. Comparative photosynthetic production of Mohave Desert shrubs. Ecology 56:732-736.

Beatley, Janice C. 1965. Ecolngy of the Nevada Test Site. II. Status of introduced species. USAEC Report UCLA 12-554. 39 p.

Beatley, Janice C. 1976. Vascular plants of the Nevada Test Site and central-southern Nevada. TID-26881, N.T.I.S., U. S. Dep. of Commerce Springfield, Va. 22161. 308 pp.

Best, J. A. 1974. The effect of soil pH on s-triazine dissipation using a balance sheet approach. Diss. Abstr. Int. 34:4155B.

Best, J. A., J. B. Weber, T. J. Monaco. 1975. Influence of soil pH on s-triazine availability to plants. Weed Sci. 26:378-382.

Birk, L. A., and F.E.B. Roadhouse. 1964. Penetration of and persistence in soil of the herbicide atrazine. Can. J. Plant Sci. 44:21-27.

Buchanan, G. A., and A. E. Hiltbold. 1973. Performance and persistence of atrazine. Weed Sci. 21:412-416.

Dowler, C. C., W. Forester, and F. H. Tschirley. 1968. Effects and persistence of herbicides applied to soil in Puerto Rican forests. Weed Sci. 16:45-50.

Eckert, Richard E., Jr. 1974. Atrazine residue and seedling establishment in furrows. J. Range Manage. 27:55-56.

Fink, R. J., and O. H. Fletchall. 1969. Soybean injury from triazine residues. Weed Sci. 17:35-36.

Hance, R. J. 1973. The effect of nutrients on the decomposition of the herbicides atrazine and linuron incubated with soil. Pest. Sci. 4:817-822. Pest. Abstra. 74-1434.

Hance, R. J. 1974. Soil organic matter and absorption and decomposition of herbicides atrazine and linuron. Soil Biol. Bioch. 6:39-42.

Harris, C. I., E. A. Woolson, and B. E. Hammer. 1969. Dissipation of herbicides at three soil depths. Weed Sci. 17:27-31.

Houston, W. R. 1977. Species susceptibility to atrazine herbicides on shortgrass range. J. Range Manage. 30:50-53.

Kaufman, D. D., and P. C. Kearney. 1970. Microbial degradation of striazine herbicides. Residue Reviews 32:235-265.

Maza, B. G., and F. B. Turner. 1976. Abiotic. p. 10-15. In: F. B. Turner (ed.), Rock Valley validation site report. US/IBP Desert Biome Res. Memo. 76-2.

Muller, W. H., and C. H. Muller, 1956. Association patterns involving desert plants that contain toxic products. Amer. J. Bot. 51:825-827.

Nikolova, G. 1973. Decomposition of triazine herbicides. Selskostop. Nauka 12:67-70. Pest. Abstr. 74-1316.

Ritter, W. F., H. P. Johnson, W. G. Lovely, and M. Molnau. 1974. Atrazine, propachlor, and diazinon residues on small agricultural watersheds-runoff losses, persistence, and movement. Environ. Sci. Tech. 8:38-42.

Romney, E. M., V. Q. Hale, A. Wallace, O. R. Lunt, J. D. Childress, H. Kaaz, G. V. Alexander, J. E. Kinnear, and T. L. Ackerman. 1973. Some characteristics of soil and perennial vegetation on northern Mohave Desert areas of the Nevada Test Site. USAEC Report UCLA 12-916. 340 p.

Roeth, F. W., T. L. Lavy, and O. C. Burnside. 1969. Atrazine degradation in two soil profiles. Weed Sci. 17:202-205.

Shimabukuro, R. H., V. J. Masteller, and W. C. Walsh. 1976. Atrazine injury: Relationship to metabolism, substrate level, and secondary factors. Weed Sci. 24:336-340.

Skipper, H. O., and V. V. Volk. 1972. Biological and chemical degradation of atrazine in three Oregon soils. Weed Sci. 20:344-347.

Turner, F. B., B. G. Maza, H. W. Kaaz, and A. T. Vollmer. 1975. Abiotic. p. 11-21. In: F. B. Turner (ed.), Rock Valley validation site report. US/IBP Desert Biome Res. Memo. 75-2.

Wallace, A., V. Q. Hale, G. E. Kleinkopf, and R. C. Huffaker. 1971. Carboxydismutase and phosphoenolpyuvate carboxylase activities from leaves of some plant species from the northern Mohave and southern Great Basin Deserts. Ecology 52:1093-1095.

Wallace, A., and E. M. Romney. 1972. Radioecology and ecophysiology of desert plants at the Nevada Test Site. USAEC Report TID-25954. $439 \mathrm{p}$.

Wallace, A., and E. M. Romney. 1974. Feasibility and alternate procedures for decontamination and post treatment management of Pu-contaminated areas of Nevada. USAEC Report UCLA 12-973. 90 p.

Wallace, A., and E. M. Romney. 1976. Initial land reclamation procedures related to possible Pu-cleanup activities at the Tonopah Test Range. USERDA Report UCLA 12-1054. 15 p. 